\title{
TOMOGRAFIA COMPUTADORIZADA DE FEIXE CÔNICO COMO FERRAMENTA NA IDENTIFICAÇÃO DE CANAIS ATRÉSICOS EM ENDODONTIA: RELATO DE CASO
}

\author{
CONE BEAM COMPUTED TOMOGRAPHY AS A TOOL FOR THE \\ IDENTIFICATION OF ATRESIC CANALS IN ENDODONTICS: CASE REPORT
}

\author{
Pablo Andrés Amoroso Silva* \\ Vitor Manoel Sespede dos Santos* \\ Gustavo Nascimento de Souza Pinto** \\ Guilherme Saintive Cardia*** \\ Elen de Souza Tolentino ${ }^{\ldots+\ldots+\cdots}$
}

\section{RESUMO}

Paciente, gênero feminino, 53 anos, compareceu ao consultório para realizar tratamento endodôntico do dente 25 por indicação protética. O tratamento foi realizado e, na radiografia periapical, apenas um canal foi observado. Após 2 meses do tratamento endodôntico, a paciente relata sensibilidade na região, mas sem sintomatologia dolorosa. Ao exame clínico, observou-se uma fístula. Devido à suspeita de fratura, um exame de Tomografia Computadorizada de feixe cônico foi indicado, pelo qual observou-se que havia outro canal atrésico por vestibular, o que justificava a queixa da paciente. A conduta do endodontista foi obturar o outro canal, não havendo necessidade de retratamento do canal obturado previamente. O caso evoluiu com sucesso depois desse procedimento.

DESCRITORES: Tomografia Computadorizada de feixe cônico • Endodontia • Dente pré-molar.

\section{ABSTRACT}

Female, 53 years old, attended the clinic to perform a root canal treatment of tooth \#25 by prosthetic indication. Treatment of a single root canal was performed, and confirmed with periapical radiography. After 2 months, the patient began to complain of sensitivity in the region, but without pain. On clinical examination a fistula on the treated tooth was found. Due to a suspected of fracture, a Cone Beam Computed Tomography examination was indicated, in which it was observed another atresic root canal on buccal region, which justified the complaint of the patient. The second root canal was treated without need for retreatment of the previously obturated canal. The case evolved successfully after this procedure.

DESCRIPTORS: Cone Beam Computed Tomography • Endodontics • Premolar.

\footnotetext{
* Doutorando em Endodontia pela Faculdade de Odontologia de Bauru (FOB-USP) pablo_amoroso@hotmail.com

** Aluno do Curso de Especialização em Radiologia Odontológica do Instituto Ravel de Ensino Superior, Maringá, PR, Brasil vitorsespede@hotmail.com

*** Aluno do curso de graduação em Odontologia na Unicesumar, Maringá, PR, Brasil nsouzagustavo@gmail.com

**** Doutorando em Dentística/Materiais Dentários pela Faculdade de Odontologia de Bauru (FOB-USP) gcardia@hotmail.com

***** Professora doutora do Departamento de Odontologia da Universidade Estadual de Maringá e da Unicesumar, Maringá, PR, Brasil elen_tolentino@ hotmail.com
} 


\section{N T RODUÇÃO}

O conhecimento da anatomia interna de dentes que terão seus condutos radiculares tratados endodonticamente é de fundamental importância para o sucesso do tratamento e do prognóstico desses dentes. Essa anatomia pode se manifestar em diversas variações morfológicas. Canais supranumerários e atrésicos podem ser descobertos com o auxílio de Tomografias Computadorizadas de Feixe CôniCo (TCFC).

O grupo dentário referente aos pré-molares possui a maior diferenciação anatômica de canais radiculares, o que dificulta uma execução bem sucedida do tratamento endodôntico. Diversas variações da anatomia interna do sistema de canais radiculares podem ser encontradas, como a presença de 1,2 ou 3 canais distintos, se unindo durante o trajeto até o nível apical ou se mantendo em caminhos distintos, apresentando foraminas apicais separadas ou outras variações ${ }^{1}$.

O tratamento endodôntico desses dentes consiste no correto preparo biomecânico e na obturação dos condutos, removendo completamente o tecido vivo ou necrosado, eliminando a infecção microbiana no sistema de canais radiculares e / ou a área perirradicular e suprimindo a sintomatologia presente, devolvendo ao paciente o conforto ${ }^{2}$. Consequentemente, o clínico deve assumir que a complexida- de anatômica ocorre com frequência suficiente para ser considerada normal ${ }^{3}$.

Exames radiográficos convencionais são limitados a uma visualização bidimensional. As informações essenciais da anatomia tridimensional dos dentes e estruturas adjacentes são obscurecidas mesmo com as melhores técnicas de paralelismo ou de dissociação, sendo que distorções e sobreposição das estruturas dentárias são inevitáveis.

A grande vantagem da TCFC está na precisão geométrica tridimensional que proporciona. Por exemplo, a possibilidade da visualização das raízes dos dentes posteriores superiores e seus tecidos periapicais nos três planos ortogonais sem sobreposição do osso zigomático, do osso alveolar e das raízes adjacentes e na identificação de forames nasopalatinos ampliados, de canais radiculares, de pequenas lesões periapicais que radiograficamente não são visíveis e na avaliação de reabsorções ósseas.

O objetivo deste trabalho consiste em relatar um caso clínico de tratamento endodôntico de um pré-molar superior que apresentou um canal vestibular atrésico, o qual foi localizado por meio do exame de TCFC, permitindo assim o tratamento do mesmo, removendo a sintomatologia dolorosa que o paciente apresentava.

\section{RELATO DE CASO CLÍNICO}

Paciente de gênero feminino, 53

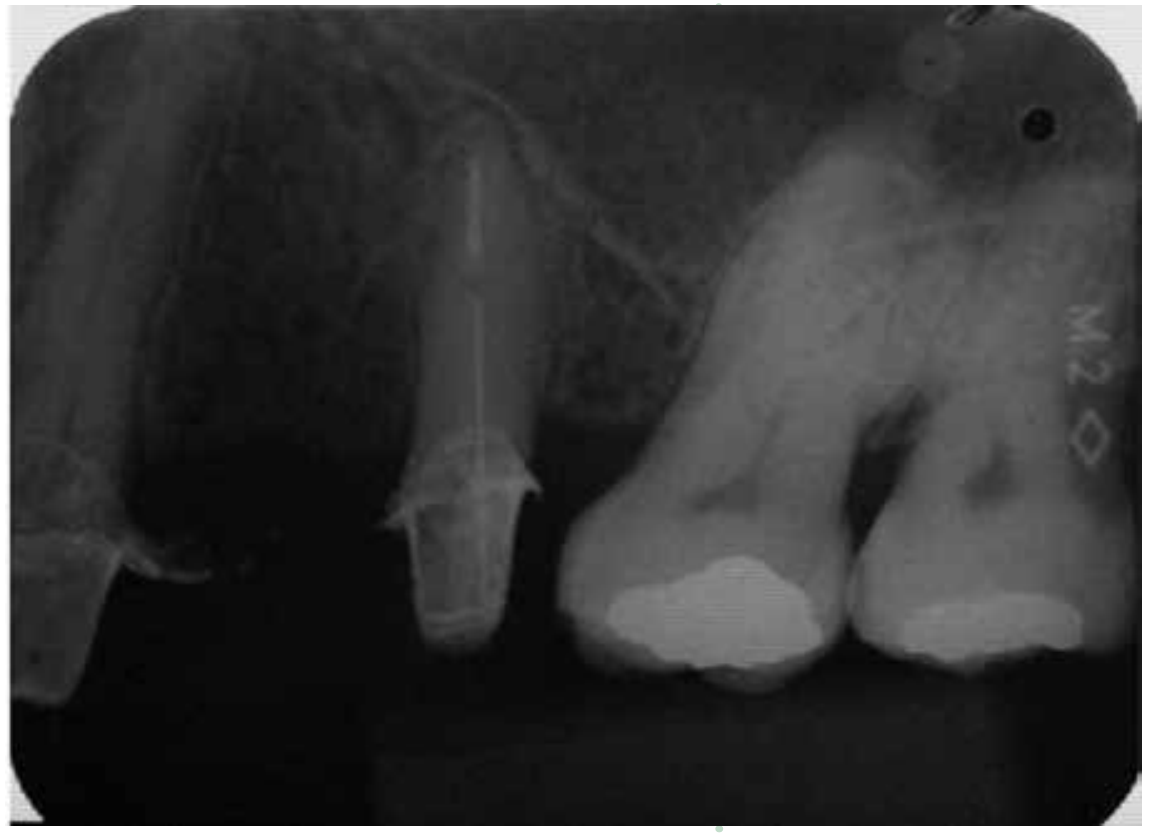

Figura 1.

Tratamento Endodôntico finalizado com núcleo cimentado e preparo protético confeccionado.
SILVA PAA

SANTOS VMS

PINTO GNS

CARDIA GS

tolentino es

TOMOgRAFIA COMPUTADORIZADA DE FEIXE CONNICO COMO FERRAMENTA NA IDENTIFICAÇÃO DE CANAIS ATRÉSICOS EM ENDODONTIA: RELATO DE CASO 
SI LVA PAA

SANTOS VMS

PINTO GNS

CARDIA GS

TOLENTINO ES

TOMOGRAFIA COMPUTADORIZADA DE FEIXE CONICO

COMO FERRAMENTA NA IDENTIFICAÇÃO

DE CANAIS

ATRÉSICOS EM

ENDODONTIA: RELATO DE CASO

172 anos, compareceu a consultório particular para realizar o tratamento endondôntico do dente 25, por indicação protética. Na exploração clínica e radiográfica apenas um canal foi observado. $\mathrm{O}$ tratamento endodôntico foi realizado (Figura 1) e, posteriormente, a paciente foi encaminhada para o protesista para a confecção de núcleo e coroa.

Após dois meses do tratamento endodôntico, já com o pino intracanal e o provisório adaptados, a paciente começou a queixar-se de sensibilidade na região, mas sem sintomatologia dolorosa intensa. Ao exame clínico, observou-se uma fístula, que foi rastreada (Figura 2). Devido à suspeita de fratura radicular, um exame de TCFC foi indicado. Na TCFC, observou-se que havia um canal adicional atrésico por vestibular, não tratado, o qual provocava essa sensibilidade, o que justificava a queixa da paciente (Figura 3 e 4).

A conduta do endodontista foi remover o pino intracanal, preparar biomecanicamente e obturar o canal vestibular atrésico, não havendo necessidade de retratamento do canal obturado previamente. O caso evoluiu com sucesso depois desse proce-

Figura 2.

Rastreamento de fístula.
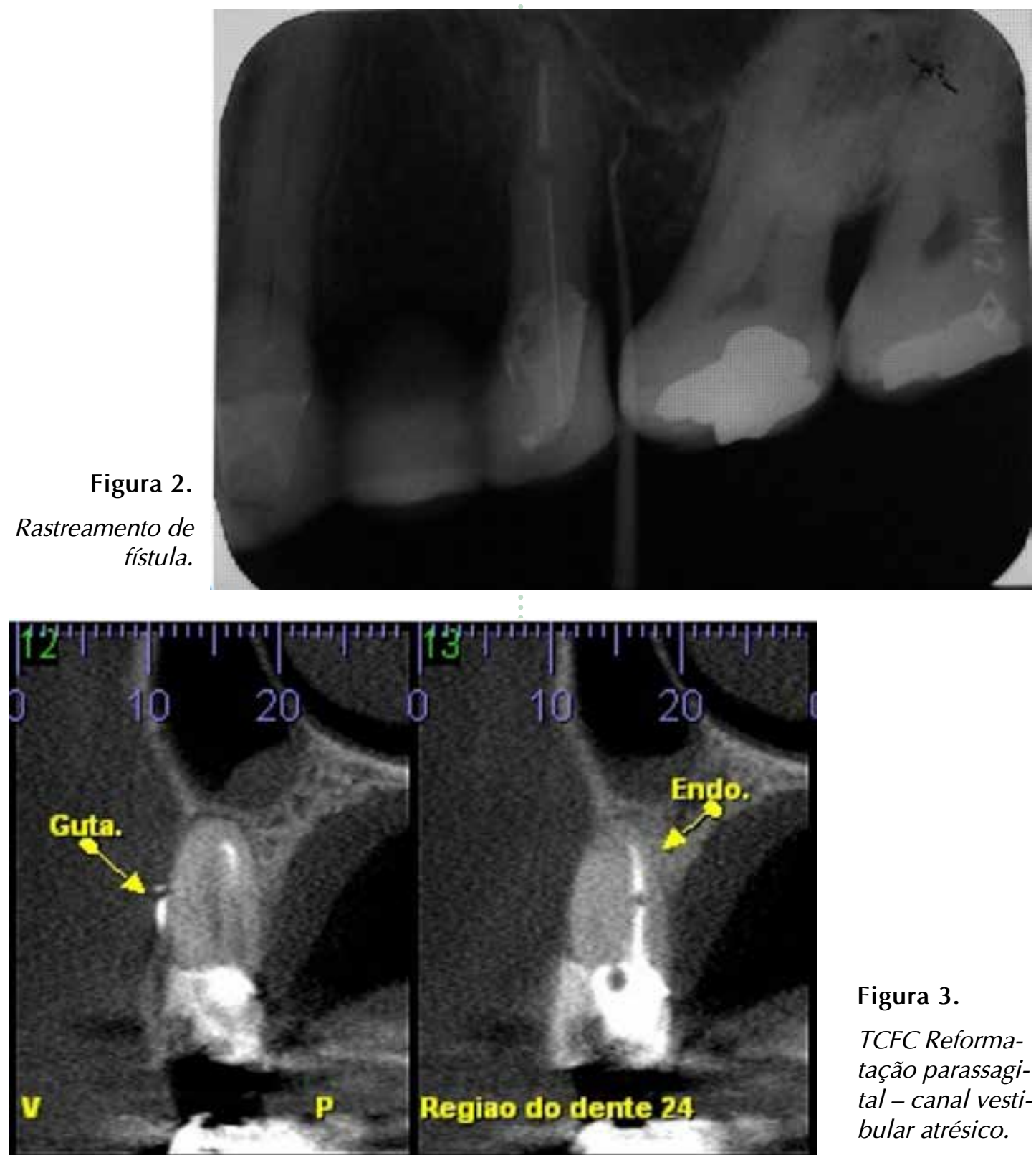

Figura 3.

TCFC Reformatação parassagital - canal vestibular atrésico. 

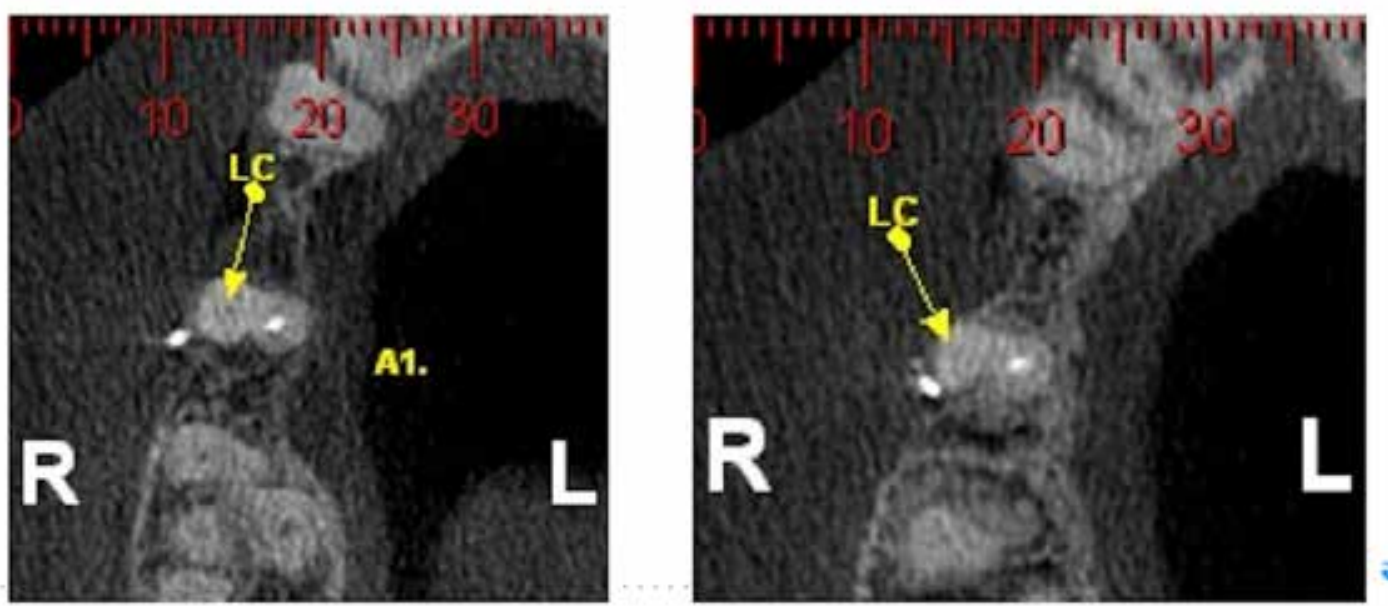

SilVA PAA

SANTOS VMS

PINTO GNS

CARDIA GS

tolentino es

TOMOGRAFIA

COMPUTADORIZADA

DE FEIXE CONICO

COMO FERRAMENTA

NA IDENTIFICAÇÃO

DE CANAIS

ATRÉSICOS EM

ENDODONTIA:

RELATO DE CASO

Figura 4. TCFC Reformatação axial - Nota-se a luz do canal vestibular (LC).

dimento, com a regressão da fístula e trazendo novamente conforto para a paciente (Figura 5), que foi reabilitada. A paciente encontra-se em proservação há 6 meses, sem queixas clínicas.

\section{I SCUSSÃ 0}

A anatomia interna do sistema de canais radiculares tem sido estudada devido a sua alta complexidade e presença de variações anatômicas, fato que influencia diretamente no sucesso da terapia endodôntica. A alta incidência de anomalias nos pré-molares superiores permanentes é confirmada na literatura e, quando presente, pode tornar o tratamento endodôntico convencional complicado, sendo necessário um cuidadoso exame clínico de exploração do canal radicular, além de uma análise detalhada do exame radiográfico, a fim de identificar algum tipo de variação.

Em muitos casos o exame radiográfico periapical pode não ser suficiente no diagnóstico por completo, como no caso citado, em que foi solicitado um exame de TCFC, no qual foi localizado o canal atrésico por vestibular. Por tratar-se do segundo pré-molar superior, que frequentemente apresenta um canal radicular único, a falha no tratamento ocorreu. A radiografia periapical não acusou um segundo canal, assim como na abertura coronária a segunda embocadura também não foi visualizada. Previamente à tomografia, a técnica de Clark foi realizada, não obtendo resultados que contribuíssem no diag-

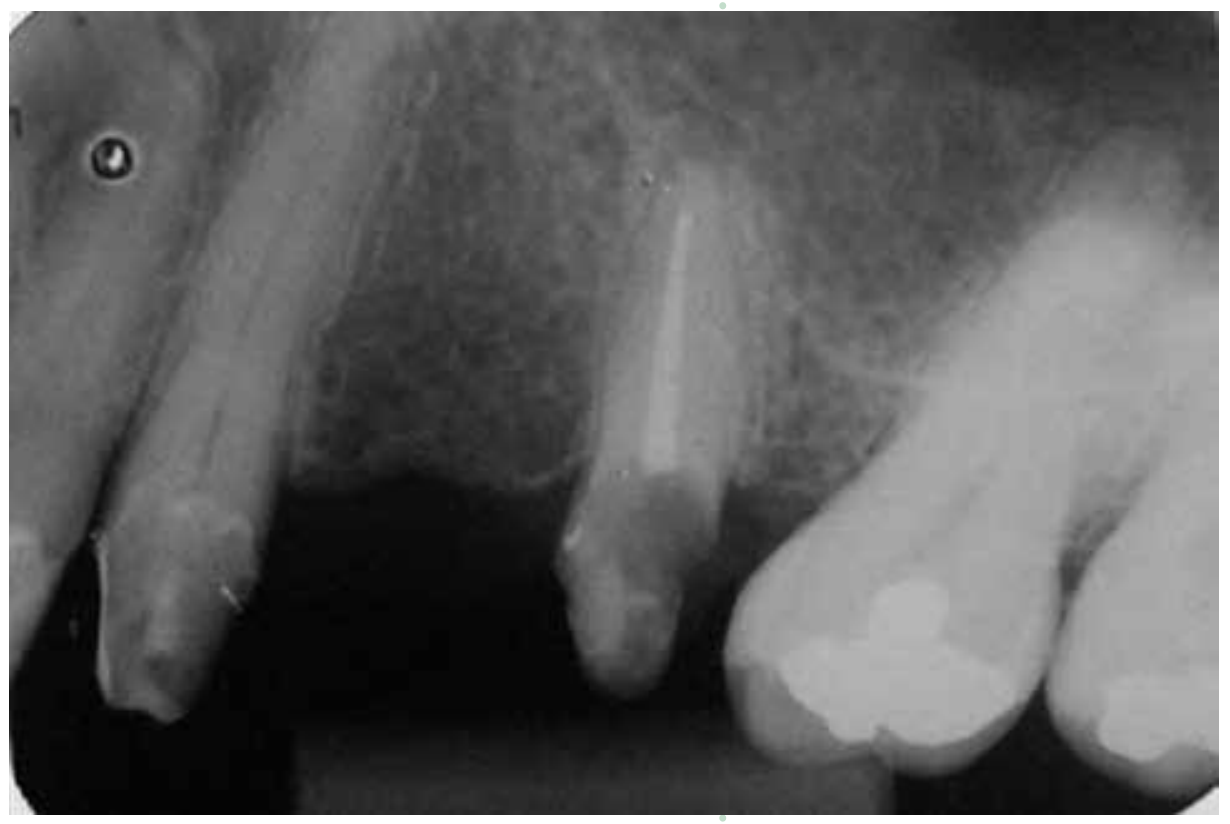

Figura 5.

Radiogra-

fia após

tratamento

endo-

dôntico

do canal

vestibular

e evolução

do caso. $\because 173 \ldots$

REV, ODONTOL

UNIV, CID, SÃo

PAULO

2013; $25(2): 171$ -

6 , MAIO-AGO 
SILVA PAA

SANTOS VMS

PINTO GNS :

CARDIA GS

TOLENTINO ES

TOMOGRAFIA COMPUTADORIZADA DE FEIXE CONICO COMO FERRAMENTA NA IDENTIFICAÇÃO DE CANAIS

ATRÉSICOS EM ENDODONTIA: RELATO DE CASO

\section{4}

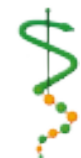

REV, ODONTOL.

UNIV, CID, SÃO

PAULO

2013; 25(2): $170-$

5, MAIO-AGO
I SSN 1983-5183

nóstico. Outro "obstáculo" no presente caso foi encontrado após o diagnóstico do canal vestibular. Questões como 'Remover ou não o pino intracanal?', 'Indicar uma cirurgia paraendodôntica com obturação retrógrada?', 'Extrair o dente uma vez que o risco de fratura é considerável?' foram discutidas entre o endodontista, o protesista e o radiologista. Com o consentimento da paciente, a opção escolhida foi a remoção do pino com auxílio do ultrassom, acesso ao canal e obturação, sem retratamento do canal palatino, mesmo conhecendo-se o alto risco desse procedimento quanto à fratura radicular.

Entretanto, algumas questões permaneceram: 'É viável ou recomendável aguardar um tempo pós-tratamento endodôntico para que a prótese seja definitivamente cimentada?', 'Quanto tempo é necessário para assegurar o sucesso do tratamento endodôntico'? No caso relatado, a paciente relatou desconforto após 2 meses do tratamento endodôntico, momento no qual a prótese fixa já estava cimentada, o que dificultou os passos posteriores. A única questão que foi esclarecida com base nessa paciente foi a de que a TCFC surge como uma ferramenta valiosa na rotina do endodontista.

Um estudo 4 comparou, ex vivo, radiografias digitais com a TCFC na detecção do número de canais radiculares em setenta e dois dentes extraídos. Os autores observaram que em $40 \%$ dos dentes os endodontistas falharam na identificação de pelo menos um canal. As imagens da TCFC proporcionaram a visualização de um maior número de canais radiculares quando comparadas às radiografias digitais ${ }^{4}$.

Alguns softwares para tomografias computadorizadas adicionam cor intensificando as características para diferenciar lesões patológicas de estruturas anatômicas normais. Imagens de TCFC têm a capacidade de mostrar fatias de um determinado tecido, com cada espessura e localização da fatia escolhida pelo operador. Por essa razão, a TCFC pode possibilitar ao operador a análise de múltiplas fatias de raízes dentárias incluindo seu sistema de canais radiculares. Esse exame radiográfico tem sido sugerido como a modalidade de ima- gem preferencial em situações difíceis que demandam localização e descrição do sistema de canais radiculares, devido à sua habilidade de fornecer informações 3D 4 .

Em função de sua natureza bidimensional, somente pela radiografia convencional é difícil a determinação do número exato de canais radiculares presentes em um dente, mesmo que ampliada, tornando o tratamento mais imprevisível ${ }^{5}$. Com a tomografia computadorizada, a morfologia radicular e a topografia óssea podem ser visualizadas em três dimensões, assim como o número de canais radiculares ${ }^{6}$.

O uso da TCFC possibilita visualizar a morfologia radicular dos canais em três dimensões, assim como o número de canais, os pontos de convergência e divergência e o seu tamanho. Canais não tratados ou com tratamento insatisfatório também são visualizados ${ }^{7}$. Segundo Cotrim-Ferreira $(2008)^{8}$, essa metodologia é mais simples e oferece menor dose de radiação do que a TC helicoidal, menor tempo de aquisição, maior possibilidade de trabalhar as imagens e obtenção de imagens na proporção 1:1, a partir de reconstruções que podem ser sequenciais, com a resolução de cortes de $1 \mathrm{em} 1 \mathrm{~mm}$ do objeto ${ }^{8}$.

Essa tecnologia comandada pelo cirurgião-dentista traz avanço para a radiologia odontológica, por permitir a visualização de estruturas de dimensões reduzidas com menor exposição à radiação para o paciente quando comparada à TC Helicoidal. Entretanto, é importante enfatizar que a dose de radiação da TCFC é maior que a das radiografias convencionais e que, apesar de suas inúmeras vantagens, seu uso não deve ser indiscriminado. Esse fato justifica a indicação da técnica de dissociação de raízes (Clark) em um primeiro momento no caso relatado. O custo de ambos os exames também deve ser levado em consideração em determinadas circustâncias.

A TCFC é um exame para diagnóstico diferencial que pode ser solicitada por cirurgiões-dentistas que encontram dificuldades em certas anatomias radiculares, ou em casos de tratamento insatisfatório com presença de dor pós-operatória e lesões periapicais sem causa aparente. 


\section{CONCLUSÃO}

A TCFC é um importante recurso auxiliar para a prática endodôntica, principalmente em casos complexos, como a localização de canais radiculares atrésicos, identificação de patologias, reabsorções e

\section{REFERÊNCIAS}

1. Vertucci FJ. Root canal anatomy of the human permanent teeth. Oral Surg Oral Med Oral Pathol 1984 Nov;58(5):589-99.

2. Siqueira Junior F, Venturim K. Infecção periapical: como provável causa de insucesso endodôntico RGO 1997 maio.-jun.;45(3):152-4.

3. Cohen S, Hargreaves KM. Caminhos da polpa. 9 ed. São Paulo: Elsevier Brasil; 2007.

4. Matherne RP, Angelopoulos C, Kulild JC, Tira D. Use of Cone-Beam Computed Tomography to Identify Root Canal Systems In Vitro. Journal of endodontics 2008 34(1):87-9.

5. Patel S. New dimensions in endodontic imaging: Part 2. Cone beam computed tomography. Int Endod J 2009 Jun;42(6):463-75. fraturas radiculares. Entretanto, é importante enfatizar que a dose de radiação da TCFC é maior que a das radiografias convencionais e que, apesar de suas inúmeras vantagens, seu uso não deve ser indiscriminado.

6. Costa CCA, Moura-Netto C, Koubik ACGA, Michelotto ALC. Aplicações clínicas da tomografia computadorizada cone beam na endodontia. Rev Inst Ciênc Saúde 2009 27(3):279-86.

7. Almada IR. Cone Beam em endodontia [Dissertação]. Porto: Universiade Fernando Pessoa; 2011.

8. Cotrim-Ferreira F, Lascala CA, Costa C, Garib DG, Chilvarquer I, Cavalcanti MGP, et al. Modernos métodos de radiologia e imaginologia para o uso ortodôntico. OrtodontiaSPO 2008 41(1):62-71.

Recebido em 05-07-2013

Aceito em 19-06-2013
SILVA PAA

SANTOS VMS

PINTO GNS

CARDIA GS

TOLENTINO ES

TOMOGRAFIA

COMPUTADORIZADA

DE FEIXE CONICO

COMO FERRAMENTA

NA IDENTIFICAÇÃO

DE CANAIS

ATRÉSICOS EM

ENDODONTIA:

RELATO DE CASO

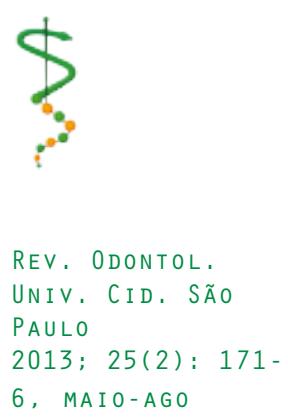

